\title{
EMERGENCY ABDOMINAL SURGERY;
} INCIDENCE OF INTRA-ABDOMINAL SEPSIS AND ITS MANAGEMENT

DR. JUNAID SULTAN, M.B.B.S

Medical Officer

Shifa International NA- 397-B, 7th Road

Hospital Satellite Town, Islamabad, Rawalpindi

DR. HARIS BIN BILAL, M.B.B.S

House Officer

Cardiac Surgery Unit London Chest Hospital

England

\section{DR. HUMAIRA KIRAN, M.B.B.S, FCPS-I \\ Medical Officer \\ Pathology Department Queen Elizabeth Hospital \\ Kingslynn, England}

\author{
Dr. Badar Bin Bilal, M.B.B.S, MRCP-I \\ Medical Officer \\ Medical Unit Old Church Hospital \\ Romford, Esser, England
}
Prof Dr. Azam Yusuf, M.B.B.S, FCPS, FRCS
Head of Surgical Unit
Rawalpindi General Hospital
Rawalpindi

\begin{abstract}
Intra-abdominal (I.A) sepsis is widely recognized as the leading cause of death in patients following emergency abdominal surgery. Objective To determine the incidence of intra-abdominal sepsis after emergency abdominal surgery. To study the predisposing factors which lead to post-op. I.A sepsis. To determine the micro flora involved in I.A sepsis Study design: A quantitative cross sectional study.. Place of study: Surgical unit-l General Hospital Rawalpindi. Period: March 1999 to August 2000. Material \& methods:565 patients) were followed after emergency abdominal surgery and incidence determined. Results: These 565 patients included 400 patients of acute appendicitis, 140 patients of emergency laparotomy and 25 patients of obstructed/strangulated hernias. The incidence of I.A sepsis after emergency abdominal surgery was found to be $10.6 \%$ with incidence of generalized I.A sepsis of $6.6 \%$. Different risk factors predispose to the development of post-op. I.A sepsis. Aerobic microflora of the I.A sepsis consists predominantly of E.coli. Overall mortality rate was $7.6 \%$ with $4 \%$ cases due to post-op. I.A sepsis. Mortality rate in patients with post-op. I.A sepsis was $38.33 \%$ with mortality rate of $78 \%$ in patients with post-op, generalized I.A sepsis and of $20 \%$ in patients with I.A abscesses. Conclusion: IA sepsis continues to be one of the major challenges in general surgery. A successful outcome depends on early diagnosis, rapid and appropriate surgical intervention, prompt administration of broad spectrum antibiotics and aggressive supportive care in ICU remains the therapeutic strategy for these patients. It is important first to determine the risk status of these patients and then to apply proper and sound surgical technique and do timely decision.
\end{abstract}




\section{INTRODUCTION}

Intra-abdominal (LA.) infections have been a continuous therapeutic and diagnostic challenge. Until the end of the last century, LA infections were treated non-surgically and the mortality was $90 \%$. The prognosis has improved recently as a result of earlier recognition by the clinician and improvement in imaging techniques. Published mortality rates are still at least $30 \%$ and morbidity is substantial ${ }^{1}$.

The incidence of LA sepsis is $10-15 \%$ after emergency abdominal surgery. It may occur as a result of contamination that occurred at the time of initial operation, anastomotic leak, iatrogenic perforation of hollow viscus, presence of foreign body, technical error compromising the vascularsupply of an anastomosis. LA hematoma may become secondarily infected or in a critically ill patient from failure of barrier function of gut with translocation of intra-Luminal organisms in to the peritoneal cavity ${ }^{2}$.

Spectrum of post-op. LA sepsis varies from an acute progressive peritonitis to chronic indolent LA abscess. Post-op peritonitis has very high mortality in the range of $50-70 \%$ even with availability of new antibiotics and good ICU facilities.

The risk of post-op I.A sepsis increases with delay in presentation, increased age, anemia, pre-existing serious underlying diseases and malnutrition. Ultrasound and CT scan are particularly effective with accuracy of about $90 \%$ in diagnosing postoperative LA abscesses and of about $50-60 \%$ for post-op generalized peritonitis. But the clinical judgment of a surgeon is most crucial in early detection of LA sepsis. Outcome depends on early diagnosis and operation. If re-laparotomy for LA sepsis is not done within $48 \mathrm{hr}$ or before the development of MODS, then mortality is very high in the range of $70-80 \%$.
The purpose of the present study was to find the incidence of post-operative LA sepsis and the predisposing factors which increased the risk of post-op sepsis, so as to recognize them earlier, and take necessary steps to decrease the incidence of post-op. LA sepsis. The study also included determining the microflora involved in LA sepsis, and the spectrum of antibiotics which could be prescribed according to culture and sensitivity.

\section{MATERIAL \& METHODS}

This study was carried out in Rawalpindi; General Hospital Rawalpindi attached with Rawalpindi Medical College. All patients in this study underwent emergency abdominal surgery from March 1999 to August 2000. A total of 565 cases were included in the study including all the emergency abdominal surgeries done during this period. These consisted of 140 cases of mid-line laparotomies, 400 cases of acute appendicitis and 25 cases of obstructed/strangulated hernias. Of the 140 cases of laparotomies, 90 patients were of acute peritonitis which included acute secondarybacterial peritonitis, traumatic peritonitis (otherforms of peritonitis) and 50 patients of acute intestinal obstruction. Of these 90 patients of generalized peritonitis, laparotomy was done in 10 patients due to gangrenous or perforated appendix with generalized peritonitis.

In 400 cases with acute appendicitis, 350 had simple acute appendicitis while 50 had gangrenous and/or perforated appendix. In all these patients localized peritonitis was present on presentation. There were 25 cases of obstructed/strangulated hernia including inguinal, umbilical, para umbilical, epigastric, incisional and femoral hernias.

The present study was a quantitative cross sectional study. In the patients in whom laparotomy was done, I/V antibiotics were given usually for period of 4-7 days depending on the underlying pathology. Injectable. 3rd generation cephalosporin with inj. 
Metronidazole was given in most of these patients where GIT pathology was involved. In cases of severe infection, injection fourth generation cephalosporins were used with Inj. Metronidazole. In cases of gangrenous or perforated appendix antibiotics were given for at least 5 days.

In all patients where diagnosis of post-operative intra-abdominal sepsis was made on clinical grounds or confirmed by ultrasound or CT scan , immediate relaparotomy was done if generalized I,A sepsis was present but if localized peritonitis or I,A abscess present, Jhen managed accordingly,

Most of the patients with absia\&SS following appendicectomy and few of patients with abscesses due to some other I.A pathology were treated conservatively with intravenous antibiotics and later converted to oral antibiotics. But if they did not improve, then early per cutaneous drainage (PCD) was done. If Pelvic abscess was present, then perrectal or per-vaginal drainage was done in these patients. In LA abscesses at other sites, PCD under ultrasound control was done. No catheter was left in place after PCD and later patient evaluated regularly by clinical signs and physical examination and by regular ultrasound examination. If residual abscess was present after PCD or recurrent, then PCD repeated, Aspirated pus was also sent for $\mathrm{c} / \mathrm{s}$ and antibiotics changed accordingly, Open surgical drainage was done when PCD was not successful,

\section{RESULTS}

Total emergency abdominal surgeries done were 565 from March 1999 to August 2000, There were 400 appendicectomies, of these gangrenous or perforated were 50. Out of 140 Laparotomies, 90 were peritonitis and 50 were of acute Intestinal obstruction. Strangulated /obstructed hernias were 25, Mean age group in appendicectomy was 10-20 $\mathrm{yr}$, in laparotomy $25-35 \mathrm{yr}$ and in hernia was $40-50$ year,
The incidence of postoperative intra-abdominal sepsis and mortality rate for each group (Appendicectomy, Laparotomy and Herniae group) are shown in table I and II, The associated causes of death were severe medical problems and other risk factors like Ml, pulmonary embolism etc, Different predisposing factors which increased the risk of post-op I A, sepsis were; Late presentation and long duration of illness, presence of MODS before operation, age $>60 y$, anemia, malnutrition, presence of pre-operative shock, presence of fecal peritonitis, presence of gangrenous gut, concomitant diseases like diabetes, CLD, CRF etc, previous history of intake of steroids, inadequate antibiotic treatment and presence of malignancy, Pus or contaminated fluid in the peritoneal cavity was sent for $\mathrm{C} / \mathrm{S}$ in 90 patients. Polymicrobial infection was present in 28 of these patients, Different bacteria were found in proportions as shown in fig 1 ,

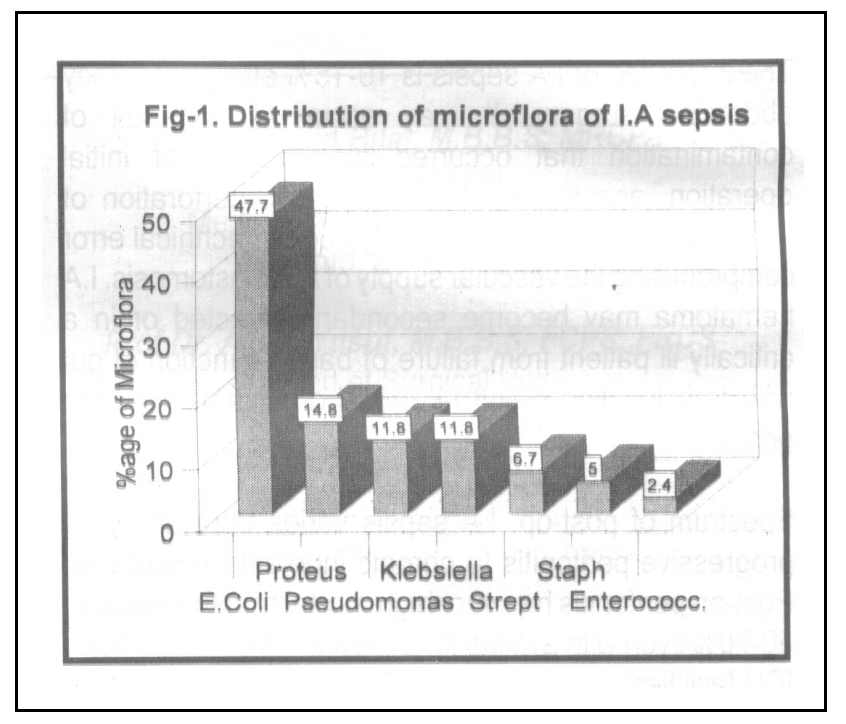

The micro flora of I.A infections was usually found sensitive to 3rd generation cephalosprins, tazobactum, Imipenam quinolones, clindamycin and amikacin, The antibiotics were used in different combinations, Overall, total patients who developed post-operative I,A sepsis after emergency abdominal surgery were $60 / 565$, Overall total 
patients expired were 40, Total patients expired due to post-op, I.A sepsis were 23 .

So Incidence of post-operative intra-abdominal sepsis after emergency abdominal surgery was $10,6 \%$, Incidence of generalized post-op I,A sepsis was $4 \%$ while incidence of post-operative LA abscess was $6,6 \%$, Over all mortality rate in patients after emergency abdominal surgery was 7,6\%, While mortality rate due to post-op I,A sepsis was $4 \%$, Mortality rate in patients who had already developed post-op LA sepsis was 38,33\%.

\begin{tabular}{|l|c|c|c|}
\hline \multicolumn{3}{|c|}{ Table-1. Incidence of I.A sepsis after emergency abdominal surgery in each group } \\
\hline Operation & Total & Generalized sepsis & I.A Abscess \\
\hline Appendicectomy & $8(2 \%)$ & $2(0.5 \%) \cdot$ & $6(1.5 \%)$ \\
\hline Lapartomy & $49(35 \%)$ & $19(13.5 \%)$ & $30(21.5 \%)$ \\
\hline Perionitis & $40(44.4 \%)$ & $15(16.6 \%)$ & $25(27.7 \%)$ \\
\hline Acute intestinal obstruction & $9(18 \%)$ & $4(8 \%)$ & $5(10 \%)$ \\
\hline Obst / strangulated hernia & $4(16 \%)$ & $2(8 \%)$ & $2(8 \%)$ \\
\hline
\end{tabular}

Table-11. Mortality rate in patients of intra abdominal sepsis

\begin{tabular}{|l|c|c|}
\hline Operation & Mortality rate & $\begin{array}{c}\text { Mortality due to IA } \\
\text { sepsis }\end{array}$ \\
\hline Appendicectomy & $4(1 \%)$ & $1(0.25 \%)$ \\
\hline Laparotomy & $32(22 \%)$ & $21(16 \%)$ \\
\hline Hernia & $4(16 \%)$ & $1(4 \%)$ \\
\hline
\end{tabular}

\section{DISCUSSION}

Incidence of intra-abdominal sepsis after emergency surgery is $12 \%$ as reported by Rinold lee Nicholas et al3. In the present study, total 565 patients were studied after emergency abdominal surgery and incidence of postoperative I.A sepsis was found to be $10.6 \%(60 / 565)$. Incidence of generalized postop. I.A sepsis was $4 \%$ and incidence of post-op. I.A abscesses was $6.6 \%$.
Overall mortality rate in patients after emergency abdominal surgery was $7.6 \%$ while mortality rate due to post-op. I.A sepsis was found to be $4 \%$. Mortality rate in patients who had developed postop. I.A sepsis was $38.33 \%$.

In patients in whom appendectomy was done, incidence of post-op. I.A sepsis was $2 \%(8 / 400)$. Incidence of generalized I.A sepsis was $0.5 \%$ while incidence of I.A abscess was $1.5 \%$. This is comparable to incidence of $1.4 \%$ after appendicectomy as study done by Reid RJ et $\mathrm{al}^{4}$.

In our study, in $85 \%$ cases of appendicectomy, appendix was found acutely inflamed and of these gangrenous and/or perforated appendices were present in 12.5 of cases. This is slightly different from study done by Reid RJ et al4 where in $80 \%$ cases, appendix was acutely inflamed and of these gang/perforated appendix was present in $17 \%$ cases. 
Mortality rate in patients who developed post-op. I.A sepsis was $12.5 \%(1 / 8)$. Overall 4 patients expired. 1 patient died due to post-op. I.A sepsis and other 3 patients expired due to associated diseases like (Ml, arrhythmias, CRF, CLD, Diabetes etc) and other related complications like anaesthesia. So overall mortality rate is $1 \%$. This is more than as study done by Wickel DJ et $\mathrm{al}^{5}$ where mortality rate was found to be $0.29 \%$. But in that study, late diagnosis of acute appendicitis was most important factor in determining the mortality but in our study high mortality was present due to associated diseases and other complications.

Mortality rate due to post-op I.A sepsis in our study was $0.25 \%$. Therefore the most important factor in these patients is found to be the presence of perforated and / or gangrenous appendix which increases the risk of post-op. I.A sepsis. All the patients who developed post-op. I.A sepsis had initially gangrenous and / or perforated appendix.

In the patients where emergency laparotomy was done, incidence of post-op. I.A sepsis was found to be $35 \%(49 / 140)$. Incidence of generalized I.A sepsis was $13.5 \%$ while of I.A abscesses was $21.5 \%$. In the peritonitis group, incidence of postoperative I.A sepsis was $44.4 \%$ with incidence of generalized I.A sepsis of $16.66 \%$ and of I.A abscesses was $27.77 \%$. This is comparable to the study done by Koperna $\mathrm{T}$ et al6 where incidence of $42 \%$ of I.A sepsis was found after laparotomy for peritonitis with incidence of diffuse I.A sepsis of $18 \%$ and $24 \%$ incidence of post-op. I.A abscesses. Incidence of post-op. I.A sepsis was found higher in patients operated for peritonitis than in those with acute intestinal obstruction. It is also found that the patients operated for traumatic peritonitis, low incidence of post-op I,A sepsis was present due to early presentation, early operation and little contamination. This is similar to study done by Behrman et al7 which showed incidence of post-op diffuse I.A sepsis to be $7 \%$ as compared to overall incidence of $17 \%$.

In the present study it is found that upper Gl perforations like DU perforation is associated with less chances of post-op I.A sepsis than patients with colonic perforations. It is also found that the leading cause of peritonitis was the perforation of hollow viscus followed by traumatic peritonitis., Percentage of patients with hollow viscus perforation was about $40 \%$ while those with traumatic peritonitis was $36 \%$. This distribution is different from the study done by Farthmann EH et al8 where they found the percentage of perforation of hollow viscus was about $60 \%$ while that of traumatic peritonitis about $20 \%$.

Due to unavailability of ICU facilities in our hospital, no treatment modalities like laparotomy or peritoneal lavage used because of lack of supportive measures. Relaparotomy was done on demand only. At initial operation, complete control of source of contamination and removal of all contaminated fluid attempted and it is found also in other studies that $90 \%$ of patients are cured at initial operation, if proper technique is used and decision taken as study done by Grunau $\mathrm{G}$ et al9 which showed that most of patients were treated with single laparotomy and intra-operative lavage.

Most useful investigation was ultrasound abdomen as study done by Khalilov AD et al10. Ultrasound showed free fluid, localized collections, loss of peristalsis and distended loops but the most important factor in the diagnosis of these patients is clinical judgement of the surgeon because in most of these patients, investigations are not helpful. So the judgment to re-operate is a critical one and attitude of the surgeon is central to this. In our study 9 patients who developed generalized post-op I.A sepsis but no relaparotomy was done and later they expired belonged to the group where investigations were not conclusive and relaparotomy delayed until MODS developed and they finally expired. In rest 
of the 10 patients with diffuse I.A sepsis where relaparotomy was done, 4 of the patients who recovered had their relaparotomy done within $48 \mathrm{~h}$ after initial operation before any evidence of MODS. Rest of the 5 patients who expired after relaparotomy, decision to re-operate was made late after 3-4 days of initial operation and when features of MODS were present and finally they expired. So early relaparotomy before the development of MODS is very important in determining the fate of patients with post-op I .A sepsis as study done by Golub R et al11 and Grunav $\mathrm{G}^{9}$.

Different factors predisposing to the development of postoperative IA sepsis are; duration of illness, presence of MODS, age $>60 \mathrm{yrs}$, anemia, malnutrition, shock, fecal peritonitis, gangrenous gut, concomitant disease, abdominal compartment syndrome, inadequate antibiotic treatment and presence of malignancy ${ }^{12,14}$. Similar factors were responsible for IA sepsis in our study.

In patients with I.A abscesses where ultrasound guided per cutaneous drainage was done and in patients with pelvic abscess where $\mathrm{P} / \mathrm{R}$ drainage was done, only single needle aspiration was done and no catheter left in place. These patients were regularly observed by clinical signs and investigations like TLC and ultrasound and regular $\mathrm{P} / \mathrm{R}$. So in all 6 patients where PCD was done, it was successful and so it had $100 \%$ success rate and only one of these patient had complication which developed bleeding from drainage site which later stopped. This is comparable to world literature ${ }^{15 " 17}$.

In 6 patients who expired with LA abscess were those in whom PCD was not possible due to presence of multiple abscesses, non availability of route, inaccessibly deep and ill - abscesses. Moreover, these patients were aged, already presence of MODS, late diagnosis, concomitant diseases and had high APACHE II score and they later expired.
To check microflora of I.A sepsis, pus and contaminated peritoneal fluid was sent for aerobic $\mathrm{C} / \mathrm{S}$ and later antibiotics changed according to $\mathrm{C} / \mathrm{S}$ report. Polymicrobial infection was found in 28 of 90 patients with ac. secondary bacterial peritonitis. E.coli was the dominant bacteria found in these patients. Percentage of different bacteria found is comparable to study done by Dietmar $\mathrm{H}$ et $\mathrm{al}^{18}$. It is also noted that in post-op I.A sepsis, spectrum of microflora found different and E.coli was not found to be the most dominant bacteria and also the rate of polymicrobial infection was found high.

The mortality rate in patients where laparotomy was done was $22 \%$. This is comparable to mortality rate of $20 \%$ in peritonitis patients as study done by Rinold Lee Nicholas et $\mathrm{al}^{3}$. Mortality rate in patients who developed post-op generalized I.A sepsis was $78 \%$ in our study which is higher than the study done by Propsescu T et al19 where mortality rate was found to be $65 \%$. Mortality rate of post-op I.A abscess was $20 \%$ in our study which was comparable to mortality rate of $19 \%$ in study done by Fry De et $\mathrm{al}^{20}$.

\section{CONCLUSION}

IA sepsis continues to be one of the major challenges in general surgery. True incidence of post op. I.A. sepsis is difficult to assess because these are most difficult patients to diagnose early and treat effectively. However, a successful outcome depends on early diagnosis, rapid and appropriate surgical intervention, prompt administration of broad spectrum antibiotics and aggressive supportive care in ICU remains the therapeutic strategy for these patients. Accurate and early knowledge of the bacteriological characteristics of flora of LA. sepsis should be obtained in all patients and antibiotics changed accordingly. Prevention is likely to be more fruitful than cure. It is therefore important to „, recognize those patients in whom the risk of post-op I.A. sepsis is more. First of all, it is important to 
determine the risk status of these patients and then to apply proper and sound surgical technique and do timely decision.

\section{REFERENCES}

1. Simon Dwerryhouse, David Leaper. Intra-abdominal and hepatic abscesses. P.106, 108. Surgery International 1996.

2. Dellinger EP, Wertz MJ, Meakins JL etal; surgical infection stratification system for intra-abdominal infection. ARCH Surg 1992: 120: 21-9.?..

3. Rinold Lee Nicholas: Intra-abdominal infection. A surgical perspective. Volume III, Issue 1-March 1996.

4. Reid RJ, Dobbs BR, Frizelle FA. Risk Factors for post-appendicectomy intra-abdominal abscess; Aust NZ J surg 1999; 69: 373-4.

5. Wickel DJ, cheadle WG, Garrison RN et.al. Poor outcome fron peritonitis is caused by disease acuity and organ failure, not recurrent peritoneal infection; Ann Surg 1997 Jun; 225(6): 744-53.

6. Koperna T, Schulz F. Prognosis and treatment of peritonitis: Do we need new scoring systems? Arch ., surg 1996; 131:180-6.

7. Behrman SW, Bertken A, Parks SN et.al Breakdown of intestinal repair after laparotomy for trauma; J Trauma 1998; 45(2): 227-31.

8. Farthmann EH, Schoffel U. Epidemiology and pathophysiology of intra-abdominal infections. Infection 1998 Sept-Oct 26 (5): 329-34.

9. Grunau G, Heemken R, Hau T. Predictors of outcome in patients with post-operative intraabdominal infection; Eur J Surg 1996; 162(8): 61925 .
10. Khalilov AD, Gadiev SI, Safarov AO. Ultrasound examination for diagnosis of post-operative peritonitis; Khirurgiia 2000; (1):28-9.

11. Golub R, Golub RW, Cantu R et.al. A multivariate analysis of factors contributing to leakage of intestinal anastomosis; J Am Coll surg 1997; 184(4): $362-72$.

12. Wickel DJ, Cheadle WG, Garrison RN et.al. Poor outcome from peritonitis is caused by disease acuity and organ failure not recurrent peritoneal infection. Ann surg 1997; 225: 744-56.

13. Bohnen JMA, mustard RA, Oxholm SE et.al. APACHE II score and abdominal sepsis. Arch surg 1988; 123:225-9.

14. Wacha H, Hau T, Ohmann $\mathrm{C}$ et.al,"Risk factors associated with intra-abdominal infections: a prospective multicenter study. Peritonitis study group; Langenbecks Arch Surg 1999 384(1): 24-32.

15. Gohl J, Gmeinwieser J, Gusinde J. Intra-abdominal .20. abscesses. Intervention versus surgical treatment; Zentralbl chir 1999; 124:187-94.

16. M.Saleem, Nadeem Ahmad, Ijaz Ahsan, Ultrasound guided percutaneous drainage of abdominal abscesses; J Pak med Assoc; 2000; 50(2): 50-54.

17. Jansen M. Truong S, Riesener KP et.al. Results of sonographically guided percutaneous catheter drainage of intra-abdominal abscesses in surgery; Chirurg 1999; 70(10): 1168-71.

18. Dietmar H. Wittmann. Intra-abdominal infections. Pathophysiology and treatment; 1991 P. 22-26.

19. Popescu I, Tublure D, Tonea A. Scheduled reinterventions in the treatment of acute peritonitis; Chirurgia1996;45:171-82.

20. Fry DE, Garrison RN: Determinats of death in patients with intra-abdominal abscess. Surgery 88 : 517.1998 . 